for care of a child with DS on PICU. A guideline was produced, edited and reviewed at a hospital guideline meeting, and agreed on by all PICU consultants.

Results Using DSMIG guidance, a hospital guideline could be produced for medical care of children with Down Syndrome, with additional specific requirements for PICU. Key PICU-specific considerations were noted and agreed on by a consultant team in terms of: airway; respiratory; cardiovascular; neurological; and infection.

Conclusion An admission to PICU can be a window of opportunity to ensure a child with DS is receiving the correct support from a multidisciplinary team, whilst there are also key PICU-specific considerations for their care. An SHO working under different leaders has an opportunity to develop a guideline to define and standardise a gold standard of care.

\section{G279(P) THE DIAGNOSIS OF ASTHMA IN CHILDREN IN PRIMARY CARE AND THE EMERGENCY DEPARTMENT}

A Oswal. Department of Paediatrics, Southend University Hospital, Southend, UK

\subsection{6/archdischild-2020-rcpch.242}

Aims This audit was undertaken to measure compliance of the diagnosis of asthma in children to NICE guidelines (Age over 5 and documented objective evidence of asthma). In the primary care audit, the aims were to review the age at diagnosis and the percentage of children in whom objective evidence of asthma was documented. In the emergency department (ED) audit, the aims were to review at the age at diagnosis, whether any previous evidence of asthma was documented, and whether the child was discharged from ED.

Methods A snapshot audit at a general practice surgery, was undertaken of children (less than 16 years old) with a coded diagnosis of 'Asthma', to review the age at diagnosis and whether any objective evidence of asthma was documented at diagnosis.

50 consecutively cases of asthma in children presenting to the emergency department (ED) in a district general hospital were then retrospectively audited to review the age at presentation, whether there was evidence of previous asthma diagnosis and whether the patient was discharged from ED.

Results In the GP surgery, there were 83 coded cases of asthma in children. 51 children (61\%) were diagnosed under 5 , with 9 children (11\%) who had objective evidence of asthma documented at diagnosis.

In the ED, 50 cases were collected of which 4 were excluded as they were referred to the other services, or for incorrect coding for unrelated diagnoses. Of the 46 remaining, 14 were under the age of $5(30 \%)$ and 27 diagnoses conformed to NICE guidelines (59\%). 33 children (72\%) were discharged from ED to their GP, 20 of whom had correct diagnoses (61\%).

The coding system in ED did not allow for separate coding of viral induced wheeze and asthma hence the 19 incorrect diagnoses likely represented viral wheeze.

Conclusions The most likely reason for the apparent low rate of correct diagnosis is the significant challenge of obtaining objective evidence of asthma in children. This audit also adds evidence to suggest that discharging children from ED with incorrectly coded diagnoses can further confound the already complex issue of diagnosing asthma in children.

\section{G280(P) BREATHING PATTERN DISORDERS: CHARACTERISTICS AND OUTCOMES OF CHILDREN ATTENDING A SECONDARY CARE RESPIRATORY CLINIC}

${ }^{1}$ TP Newson, ${ }^{2}$ A Elias. ${ }^{1}$ Paediatric Department Kent and Canterbury Hospital East Kent Hospitals NHS University FT, Canterbury, UK; ${ }^{2}$ Speech and Language Therapy Department, Canterbury and Kent Community Trust, Canterbury, UK

\subsection{6/archdischild-2020-rcpch.243}

Aim Breathing Pattern Disorders (BPD) previously termed dysfunctional breathing are due to functional abnormalities of the thoracic or laryngeal mechanics of respiration that result in inefficient breathing and a variety of symptomatology. Symptoms such as dyspnoea are significantly handicapping and disproportionate to extent and severity of any associated disease e.g. asthma. Paediatric studies of BPD and outcomes are few yet we are increasingly recognising children attending our respiratory clinic and referring patients for specialist speech and language therapy (SALT). We therefore reviewed our experience.

Methods Retrospectively we analysed health records of 18 children with BPD referred to specialist SALT between 2015 to 2018 from a respiratory clinic.

Results Age range 11-16 years (median 14 with 14 female, 4 male). Co-morbidities 16 Asthma,2 Oesophageal atresia \& TOF repair, 1Chronic Regional Pain Syndrome, 1Chronic Fatigue Syndrome. Commonest BPD was induced laryngeal obstruction (ILO) in 16 patients (exercise induced EILO in 15). Other types of BPD included hyperventilation in 7; psychogenic cough 8 and dysphonia1. A single BPD was present in 6 (EILO) with two or more in 10 patients. Inducers of BPD were exercise 16; bullying 3 ; anxiety 14 ; stress 11 ; weather 3; posture 1; odours 3. Majority had significant school absences (1-24 months). Delay in diagnosis (1-24 months median 6 months). Emergency calls in 14 with one patient with ILO needing ventilation. Follow up minimum at 1 year 2 complete resolution, 14 good control of BPD and more confident with improved morbidity and school attendance; 2 no improvement; 1 lost to follow up. De- escalation of asthma treatment in 16 patients.

Conclusion BPD are important to recognise and treat early to limit the significant morbidity that adversely impacts quality life. The establishment of a referral pathway with early input of a specialist SALT allows rapid resolution of symptoms in many and prevents exhaustive unnecessary investigation and those with co-existing asthma inappropriate escalation of treatment.

\section{G281(P) IMPROVING PARENTAL UNDERSTANDING OF CHRONICNEONATAL LUNG DISEASE (CNLD) AND SATISFACTION WITH INFORMATION PROVISION REGARDING CNLD AND HOME OXYGEN PATHWAY}

${ }^{1} \mathrm{~L}$ Jones, ${ }^{1} \mathrm{R}$ McCrum, ${ }^{1} \mathrm{~S}$ Smith, ${ }^{1} \mathrm{M}$ Bharadwaj, ${ }^{2} \mathrm{~S}$ Mulla. 'Medical education, Kings College London, London, UK; ${ }^{2}$ Neonatology, East Kent University Hospitals NHS Trust, Ashford, UK

\subsection{6/archdischild-2020-rcpch.244}

Background CNLD is a multifactorial condition affecting premature infants and remains a major source of morbidity and mortality for extremely low birth weight infants. The management of the disease is a large burden to the child's family due to its long and complicated multidisciplinary care-pathway. Therefore, it is vital to fully educate parents 